\title{
COVID-19 patients may present with myocarditis: A case report emphasizing the cardiac involvement of SARS-CoV-2
}

\author{
Ebrahim Salavati ${ }^{1}$, Hamidreza Hajirezaee $^{1}$, Hamid Reza Niazkar $^{2} * \mathbb{D}$, Muhammad Sadegh Ramezani $^{1}$, Alireza Sargazi ${ }^{3}$ \\ Received: 11 Jul 2020 \\ Published: 16 Aug 2021
}

Abstract

The Coronavirus disease 2019 (COVID-19), which was declared to be pandemic on March 12, 2020, is the latest health concern worldwide. COVID-19 patients may develop cerebrovascular complications either during the course of COVID-19 or even as an initial presentation of the disease. Herein, a case of myocarditis in a COVID-19 patient without any respiratory signs and symptoms is presented.

Keywords: COVID-19, SARS-CoV-2, Coronavirus, Myocarditis, Myopericarditis

\author{
Conflicts of Interest: None declared \\ Funding: None \\ *This work has been published under CC BY-NC-SA 1.0 license. \\ Copyright $₫$ Iran University of Medical Sciences
}

Cite this article as: Salavati E, Hajirezaee H, Niazkar HR, Ramezani MS, Sargazi A. COVID-19 patients may present with myocarditis: A case report emphasizing the cardiac involvement of SARS-CoV-2. Med J Islam Repub Iran. 2021 (16 Aug);35:104. https://doi.org/10.47176/mjiri.35.104

\section{Introduction}

The Coronavirus disease 2019 (COVID-19), which was declared to be pandemic on March 12, 2020, is the latest health concern worldwide. Its pathogen, severe acute respiratory syndrome coronavirus 2 (SARS-CoV-2), was first isolated from pneumonia patients with an unknown etiology in Wuhan, China, in December 2019. So far, COVID19 has infected more than 110,970,000 individuals and yielded more than $2,460,000$ deaths. The mortality rate of COVID-19 is estimated to be between $2 \%$ and $4 \%$. Nevertheless, individuals with preexisting medical conditions as well as elder ones are at a higher risk of mortality (1-4).

Cardiovascular diseases are known to be one of the main risk factors in a patient experiencing severe COVID-

\footnotetext{
Corresponding author: Hamid Reza Niazkar, niazkar@gmu.ac.ir

1. Bohlol Hospital, Gonabad University of Medical Sciences, Gonabad, Iran

2. Student Research Committee, Gonabad University of Medical Sciences, Gonabad, Iran

3. Dey Hospital, Torbat Heydarieh University of Medical Sciences, Torbat Heydariyeh, Iran
}

19 (2). In this regard, COVID-19 patients may also develop cerebrovascular complications either during the course of COVID-19 or even as an initial presentation of the disease. So far, various cardiac complications of COVID-19 have been reported. These complications include heart failure, arrhythmia, myocardial infarction, and myocarditis (5-7). Herein, a case of myocarditis in a COVID-19 patient without any respiratory signs is presented.

\section{Case Report}

\section{History of Presentation}

A 30-year-old man was referred to our hospital complaining of acute-onset epigastric pain, which was started

$\uparrow$ What is "already known" in this topic:

COVID-19 patients may develop cerebrovascular complications either during the course of COVID-19 or even as an initial presentation of the disease. So far, various cardiac complications of COVID-19 have been reported. These complications include heart failure, arrhythmia, myocardial infarction, and myocarditis.

\section{$\rightarrow$ What this article adds:}

In this article, a case of myocarditis in a COVID-19 patient without any respiratory signs and symptoms is presented. COVID-19 may present with myocarditis, even in the absence of noticeable respiratory involvement. In the context of the COVID-19 pandemic, physicians should be aware of viral myocarditis secondary to the SARS-CoV-2. 
three days ago. His pain was positional, relieved by leaning forward, and it was radiating to the left arm. He had no other signs except diarrhea and nausea. At the admission, his vital signs were within the normal range (oxygen saturation $\left(\mathrm{SPO}_{2}\right): 97 \%$, Respiratory Rate: 23 , Pulse Rate: 98 bpm, Blood Pressure: 100/80 mm Hg, Temperature: 37.1 degrees Celsius), and the general appearance of the patient seemed to be ill. Physical examinations revealed no abnormality.

\section{Past Medical History}

He was non-smoker with no history of heart diseases, surgeries, or any other related diseases.

\section{Differential Diagnosis}

Based on the patient's presentation, various possible differential diagnosis was made for the patient including gastrointestinal and biliary diseases, such as acute pancreatitis, peptic ulcer disease, and cardiac diseases, such as pericardial diseases and myocardial infarction. In this regard, in addition to the routine evaluations, further assessments such as liver, biliary and cardiac enzymes were carried out for the patient.

\section{Investigations}

The initial patient's electrocardiogram (ECG) revealed normal sinus rhythm, normal axis deviation, and diffuse ST-segment elevation (Fig. 1). In the laboratory investigation, the troponin test was positive $(>50 \mathrm{ng} / \mathrm{l})$, C-reactive protein was +2 . The white blood cell count was 9700 per $\mathrm{ml}$, with the 1100 absolute lymphocyte count. All other laboratory tests, including liver enzymes, were within the normal ranges. Later, echocardiography revealed a normal left ventricular size with mild systolic dysfunction, mild diastolic dysfunction, no significant valvular heart disease, low ejection fraction of $45 \%$ with global hypokinesia, and normal pulmonary artery pressure. In this regard, the myopericarditis diagnosis was made. Later, cardiac magnetic resonance imaging revealed diffuse myocardial wall edema and decreased the left ventricular ejection fraction. The serological assessments for viruses, which were responsible for myocarditis, such as Human Immunodeficiency Viruses, Epstein-Barr virus, Cytomegalovirus, hepatitis $\mathrm{B}$ and $\mathrm{C}$, were negative. In addition, immunological assays for autoimmune diseases were performed, which were found to be negative. In the context of the COVID-19 pandemic and diagnosis of viral myocarditis, a real-time reverse transcriptase-polymerase chain reaction (RT-PCR) test for COVID-19 was performed and found to be positive despite the apparent normal computed tomography (CT) of lungs (Fig. 2).

\section{Management}

For six days, the patient was prescribed ASA $650 \mathrm{mg}$ every night, and Colchicine $0.5 \mathrm{mg}$ every twelve hours along with Azithromycin $500 \mathrm{mg}$ stat and then $500 \mathrm{mg}$ daily. Afterward, the patient was discharged completely free of symptoms with an ejection fraction of $55 \%$. It should be noted that the patient did not develop any arrhythmias during admission. Also, he was completely

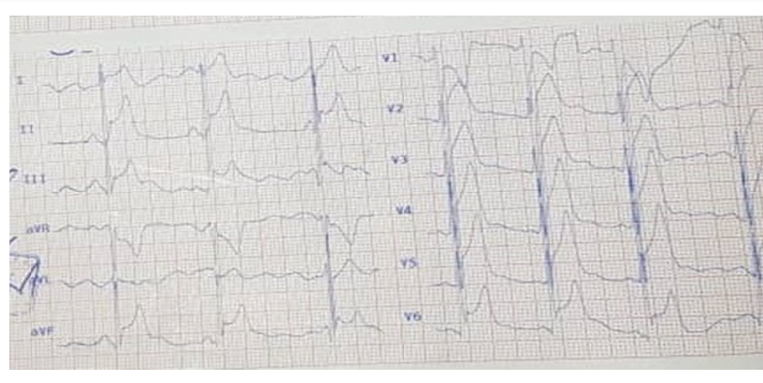

Fig. 1. The initial ECG of the patient, normal sinus rhythm, normal axis deviation, and diffuse ST elevation in lead I, II, I II, avF and $\mathrm{V}_{2}$ to $\mathrm{V}_{6}$

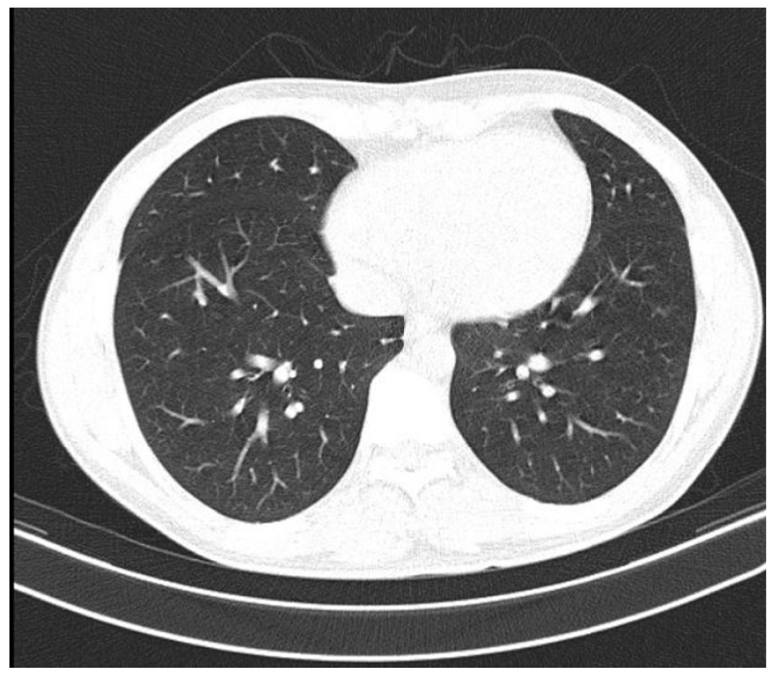

Fig. 2. The patient's spiral lung CT scan with no signs in favor of COVID-19 infection

asymptomatic after seven days of follow-up.

\section{Discussion}

In this study, a case of the COVID-19 patient without any respiratory signs and symptoms is presented. To our knowledge, this is the second reported case of myocarditis in the COVID-19 patient without any respiratory involvement (7). Our patient developed myocarditis in the absence of an evident respiratory involvement. This will unarguably emphasize the role of cardiac injury in the COVID-19 infection.

According to studies, the chromosome of SARS-CoV-2 was found to be more than $80 \%$ similar to that of the severe acute respiratory syndrome $(\operatorname{SARS})(8,9)$. In this regard, the cardiac involvement of SARS is welldocumented $(7,9)$. However, little has been known so far about the possible heart involvement of SARS-CoV-2. In addition, the increased levels of troponin, a new pathologic finding in ECG or echocardiography, were observed in more than twenty percent of severe cases of COVID-19 $(10,11)$.

Viral myocarditis, one of the main causes of myocarditis, is associated with various viruses including parvovirus, enteroviruses, adenovirus, coxsackievirus B, and human herpesvirus 6 (12). On this subject, viral myocarditis is associated with various signs and symptoms, such as 
dyspnea, fatigue, chest discomfort, palpitations, and may develop into severe heart failure or arrhythmia (13). Also, according to the literature, more than sixty percent of myocarditis patients may experience arthralgia, fever, sweating, respiratory or gastrointestinal symptoms weeks prior to the disease (14). The exact mechanism and the prevalence of myocarditis in the COVID-19 patients are still unknown (15). However, various possible mechanisms were proposed for myocardial inflammation of SARS$\mathrm{CoV}-2$, such as the indirect damage due to autoimmune reactions secondary to the cytokine storm or the direct invasion of SARS-CoV-2 through the angiotensinconverting enzyme 2 (ACE2) receptors $(11,16)$. Needless to say, further studies in the aspect of the cardiac involvement of SARS-CoV-2 are encouraged.

There are limited studies regarding COVID-19 associated with myocarditis. In addition, in most of these cases, patients were exhibiting respiratory signs and symptoms. However, our patient exhibited COVID-19 related myocarditis without any respiratory signs and symptoms. Therefore, in the context of the COVID-19 pandemic, the clinical suspicion of COVID-19 associated with myocarditis should be considered as a possible diagnosis even in those without any respiratory signs and symptoms. In such patients, the cardiac MRI and echocardiography will inevitably help physicians to diagnose (17-19).

The management of myocarditis in the COVID-19 patients is still under debate. In a study by $\mathrm{Hu}$ et al., the administration of glucocorticoids and immunoglobulin therapy was suggested in similar patients (20). However, limited data are available on the beneficial use of immunoglobulins and glucocorticoids in acute myocarditis (11, 20). Our patient recovered after six days of treatment with ASA, Colchicine, and Azithromycin.

Our study limitation includes the absence of histological assessment and endomyocardial biopsy, which is known to be the gold standard diagnostic test of viral myocarditis $(11,20)$. Since the patient refused to undergo further assessments, another limitation to our study is the absence of a coronary artery calcium scan for ruling out the coronary artery diseases.

\section{Conclusion}

COVID-19 may present with myocarditis, even in the absence of noticeable respiratory involvement. In the context of the COVID-19 pandemic, physicians should be aware of viral myocarditis secondary to the SARS-CoV-2. Such awareness will inevitably prevent un/misdiagnosis.

\section{Acknowledgment}

The authors would like to acknowledge the devoted efforts of healthcare providers worldwide during the COVID-19 pandemic.

\section{Ethics approval and consent to participate}

Informed written consent is obtained from the patient for medical procedures and anonymously publication of the article.
Conflict of Interests

The authors declare that they have no competing interests.

\section{References}

1. Giwa A, Desai A, Jagoda A. Novel coronavirus COVID-19: an overview for emergency clinicians. Emerg Med Pract. 2020;22(2):121.

2. Yang J, Zheng Y, Gou X, Pu K, Chen Z, Guo Q, et al. Prevalence of comorbidities in the novel Wuhan coronavirus (COVID-19) infection: a systematic review and meta-analysis. Int J Infect Dis. 2020.

3. Niazkar HR, Niazkar M. Application of artificial neural networks to predict the COVID-19 outbreak. Global Health Research and Policy. 2020;5(1):1-11

4. Karimi Shahri M, Niazkar HR, Rad F. COVID-19 and hematology findings based on the current evidences: A puzzle with many missing pieces. Clin Lab Haematol. 2020.

5. Kochi AN, Tagliari AP, Forleo GB, Fassini GM, Tondo C. Cardiac and arrhythmic complications in patients with COVID-19. J Cardiovasc Electrophysiol. 2020.

6. Kim I-C, Kim JY, Kim HA, Han S. COVID-19-related myocarditis in a 21-year-old female patient. Eur Heart J. 2020.

7. Inciardi RM, Lupi L, Zaccone G, Italia L, Raffo M, Tomasoni D, et al. Cardiac involvement in a patient with coronavirus disease 2019 (COVID-19). JAMA Cardiol. 2020.

8. Xia J, Tong J, Liu M, Shen Y, Guo D. Evaluation of coronavirus in tears and conjunctival secretions of patients with SARS-CoV-2 infection. J Med Virol. 2020;92(6):589-94.

9. Chen Y, Liu Q, Guo D. Emerging coronaviruses: genome structure, replication, and pathogenesis. J Med Virol. 2020;92(4):418-23.

10. Wang $\mathrm{D}, \mathrm{Hu} \mathrm{B}, \mathrm{Hu} \mathrm{C}$, Zhu F, Liu X, Zhang J, et al. Clinical characteristics of 138 hospitalized patients with 2019 novel coronavirus-infected pneumonia in Wuhan, China. JAMA. 2020;323(11):1061-9.

11. Zheng YY, Ma YT, Zhang JY, Xie X. COVID-19 and the cardiovascular system. Nat Rev Cardiol. 2020;17(5):259-60.

12. Dadashi M, Azimi T, Faghihloo E. Global study of viral myocarditis A systematic review and meta-analysis. J Acute Dis. 2020;9(1):1.

13. Ellis CR, Di Salvo T. Myocarditis: basic and clinical aspects. Cardiol Rev. 2007;15(4):170-7.

14. Magnani JW, Dec GW. Myocarditis: current trends in diagnosis and treatment. Circulation. 2006;113(6):876-90.

15. Clerkin KJ, Fried JA, Raikhelkar J, Sayer G, Griffin JM, Masoumi A, et al. Coronavirus disease 2019 (COVID-19) and cardiovascular disease. Circulation. 2020.

16. Hatami F, Valizadeh N, Ramandi MMA. Emerging mechanisms for the new coronavirus-related myocardial injury and ischemia: A review of the literature. Anatol J Cardioll / Anadolu Kardiyoloji Dergisi. 2020;24(1)

17. Naderi N, Ansari Ramandi MM, Baay M, Hosseini Z, Zanganehfar ME, Rabieie P, et al. Cardiovascular patients in COVID-19 era, a case series, an experience from a tertiary cardiovascular center in Tehran, Iran. Clin Case Rep. 2020.

18. Beşler MS, Arslan H. Acute myocarditis associated with COVID-19 infection. Am J Emerg Med. 2020;38(11):2489. e1-. e2

19. Doyen D, Moceri P, Ducreux D, Dellamonica J. Myocarditis in a patient with COVID-19: a cause of raised troponin and ECG changes. Lancet. 2020;395(10235):1516.

20. Hu H, Ma F, Wei X, Fang Y. Coronavirus fulminant myocarditis treated with glucocorticoid and hunnan immunoglobulin. Eur Heart J. 2021;42(2):206. 